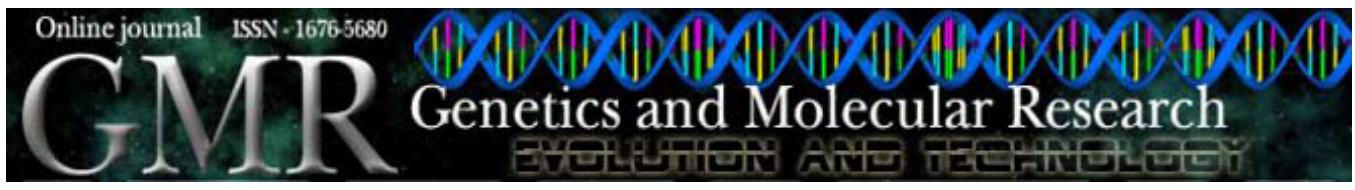

\title{
Bovine papillomavirus DNA in milk, blood, urine, semen, and spermatozoa of bovine papillomavirus-infected animals
}

\author{
C.J. Lindsey ${ }^{1}$, M.E. Almeida ${ }^{1}$, C.F. Vicari ${ }^{1}$, C. Carvalho ${ }^{2}$,

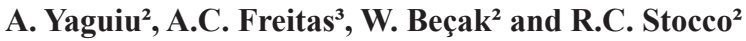 \\ ${ }^{1}$ Departamento de Biofísica, Universidade Federal de São Paulo, \\ São Paulo, SP, Brasil \\ ${ }^{2}$ Laboratório de Genética, Instituto Butantan, São Paulo, SP, Brasil \\ ${ }^{3}$ Departamento de Genética, Universidade Federal de Pernambuco, \\ Recife, PE, Brasil
}

Corresponding author: C.J. Lindsey

E-mail: lindsey@biofis.epm.br

Genet. Mol. Res. 8 (1): 310-318 (2009)

Received December 15, 2008

Accepted January 20, 2009

Published March 17, 2008

\begin{abstract}
Papillomavirus infection in bovines is associated with cutaneous papillomatosis on the hide, udders and other epithelial tissues, as well as in oral respiratory, alimentary and urinary tract mucosa. Bovine papillomavirus (BPV) is also considered the etiological agent of esophageal tumors and the malignant bladder tumors that characterize the clinical condition associated with chronic enzootic hematuria. After infective viral DNA was found in cattle blood and BPV1, 2 and 4 DNA in cattle reproductive and embryonic tissues, we looked for and found BPV DNA in blood, milk, urine, seminal fluid, and spermatozoa of BPV-infected animals. Peripheral blood lymphocyte cultures from BPV-infected animals had high rates of chromosome aberrations, including radial rearrangements
\end{abstract}


that signal oncogenic potential and viral interaction with telomeric regions. The finding of BPV DNA in body fluids and tissues other than the epithelium demonstrates co-infection of other tissues or cell types by papillomavirus and shows the potential role of lymphocytes, seminal fluid and spermatozoa in BPV transmission. Our findings reinforce a peremptory need for prophylactic and therapeutic instruments to curtail this disease in bovine livestock.

Key words: Bovine papillomavirus; Blood; Milk; Urine; Semen; Spermatozoa

\section{INTRODUCTION}

Enhancing cofactors have been associated with papillomatosis in cattle (Moura et al., 1988; Jackson et al., 1993; Stocco dos Santos et al., 1998). However, the virus itself is now considered the main etiological carcinogenic agent that produces esophagic or urinary tract tumors in bovines. It provokes chronic enzootic hematuria and/or esophagic distress, caused by bovine papillomavirus (BPV)-produced lesions, which result in high morbidity, characterized by wasting and, at advanced stages, untimely mortality (Borzacchiello and Roperto, 2008). The carcinogenic potential of papillomavirus is related to the arrest of the p53 cell cycle checkpoint. Viral-host cell chromatin interactions possibly are initiated by modification of histone conformation (Borzacchiello and Roperto, 2008), leading to viral DNA integration into host genome and/or chromosome rearrangements, most specifically those related to interaction with chromosome telomeric regions. These events are believed to be among the cellular alterations that build up to oncogenetic transformation (Borzacchiello and Roperto, 2008).

Bovine papillomavirus, as is known for other mammal-infecting papilloma viruses, is believed to selectively infect epithelial tissues, completing its biological cycle in the upper layers of the stratified epithelium (Campo, 2003), with expression of oncogenic proteins, as well the L1 and L2 capsid-forming proteins (Campo, 2003). Until now, the transmission mechanisms that have been implicated include direct frictional epithelial contact between infected individuals and contact with contaminated dairy fixtures (Campo, 2003). However, the finding that BPV infections and associated tumors can be transmitted by urinary bladder hemangiomatous lesion suspensions (Olson et al., 1959, 1965 ) and that blood from infected animals can transmit BPV2 infections to other animals and to their offspring (Stocco dos Santos et al., 1998) raises questions about the virus life cycle in peripheral blood and possible modes of vertical and horizontal transmission.

Recently, BPV2 and BPV4 DNA were identified in embryonic tissues as well as in female reproductive tissues taken from infected cows, demonstrating viral presence in tissues other than cutaneous epithelium (Yaguiu et al., 2006, 2008). Based on these initial observations, we decided to examine bovine blood, milk, urine, semen, and wart samples for BPV2 and BPV4 DNA. 


\section{MATERIAL AND METHODS}

\section{Donor selection}

Thirty-four cattle, exhibiting papillomatosis, respiratory distress and/or enzootic hematuria were randomly selected for sample collection from five heavily afflicted herds in the southeastern region (Vale do Paraíba) of the State of São Paulo, Brazil (Table 1).

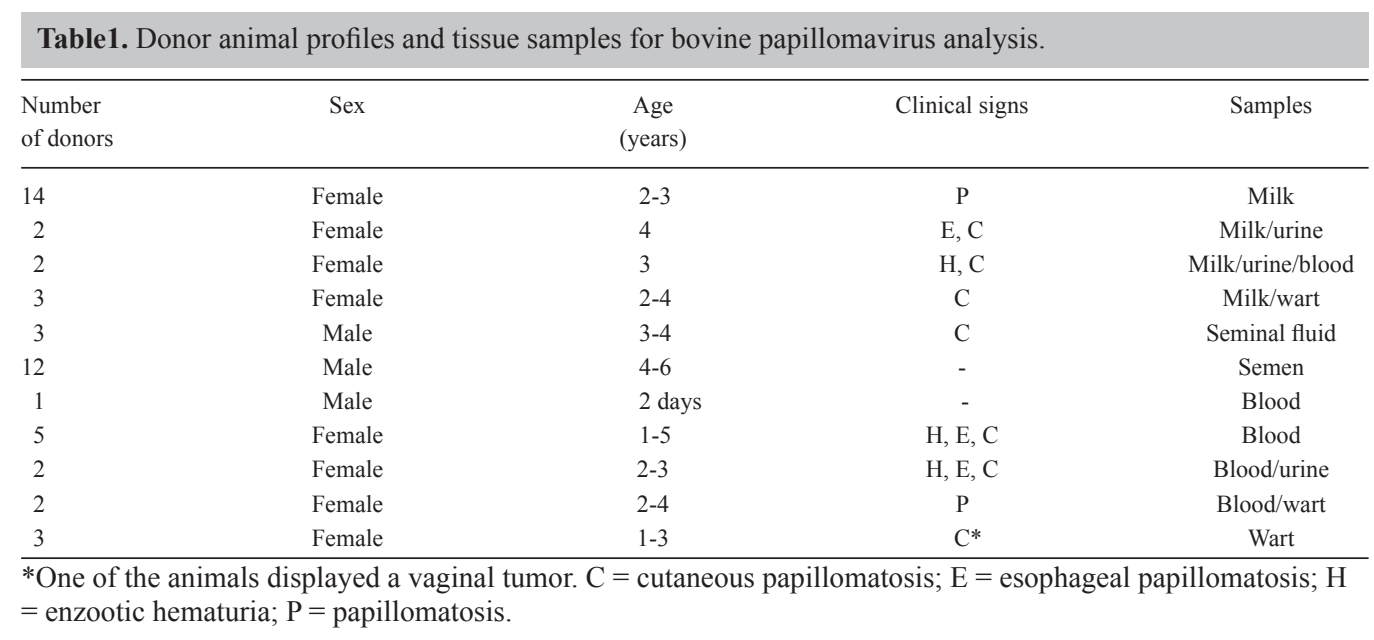

\section{Tissue and body fluid samples}

Milk, seminal fluid, urine, blood, and wart samples were collected from cattle pertaining to herds from small dairy-producing properties. The herds, ranging from 40 to 60 cattle, were BPV infested. Twelve frozen semen samples acquired from an artificial insemination commercial facility were also analyzed for BPV DNA.

\section{Sample collection}

Sterilized disposable gloves and instruments were used and changed at every step, and careful aseptic procedures were adopted to minimize sample contamination risks. Cutaneous wart samples were collected as transverse slices, made with disposable scalpels, after thorough cleansing of the cutaneous area with an iodine/alcohol antiseptic solution. The slices were immediately stored in sterile chilled sample flasks. Later, the samples were dissected to remove the vestiges of outer epithelial layer blood from the wart tissues. Urine samples were drained directly to sterile flasks from urinary bladders using sterile catheters. Milk and colostrum samples were collected directly into disposable sterile flasks after udder cleansing with antiseptic solution. Semen samples were collected directly to disposable sterile flasks after cleansing of the entire genital region. The 12 frozen semen samples were acquired from an artificial insemination commercial facility that had stored them for four years preceding analysis. The semen and seminal fluid donor animals were not available for the collection of other body fluid or tissue samples. 


\section{Sample cytological analysis}

Milk, urine, semen, and seminal fluid samples were examined for lymphocytes, epithelial cells and spermatozoa. Cytological entities in frozen semen were characterized in 3\% Giemsa solution-stained slides prepared from re-suspended and re-centrifuged $(2 \times 10 \mathrm{~min} /$ $13,000 \mathrm{rpm})$ pellets.

\section{DNA extraction, amplification and sequencing}

For the detection of BPV, $300 \mathrm{ng}$ DNA, isolated from the samples, was amplified by polymerase chain reaction (PCR) using primers specific to BPV1 (forward: 5'-ggagcgectgctaactatagga-3' and reverse: 5'-atctgttgtttgggtggtgac-3'), BPV2 (forward: 5'-gttataccacccaaagaagaccct-3' and reverse: 5'-ctggttgcaacagctctctttctc-3') and BPV4 (forward: 5'-gctgaccttccagtcttaat-3' and reverse: 5'-cagtttcaatctcctcttca-3'). These primers are complementary to the L2 and L1 regions of BPV1 and BPV2, and to the E7 region of BPV4 genes, respectively. The expected product sizes were 301, 164, and $170 \mathrm{bp}$. The amplicons were resolved either by $12.5 \%$ SDS-PAGE and visualized by silver staining (Phast system; Pharmacia Biotech) or 1\% ethidium-bromide-stained agarose gel. The frozen semen samples were also resolved on 1\% agarose gel. The sensitivity of PCR was estimated to be between 10 and 102 DNA copies per reaction. Standard procedures were used to avoid contamination.

\section{Cycle sequencing of amplified DNA}

Amplification products with 150 ng DNA were selected for the cycling reactions (Amersham RPN 2438) for BPV1, BPV2, and BPV4 and performed with labeling at the 5' end with fluorescein and run on an ALF automatic DNA sequencer (Pharmacia - Biotech).

\section{In situ hybridization}

Before in situ hybridization, the semen samples were prepared in $2 \mathrm{mmol} / \mathrm{L}$ dithiothreitol to obtain decondensed sperm heads on histological slides. In situ hybridization was done using biotin-labeled PCR products as probes. The probes, which were labeled using the GenPoint kit (Dako, Carpinteria, USA), were prepared from PCR products obtained from amplification of the cloned viral genome and from PCR products obtained from the samples. The negative controls were prepared using PCR products of genes not detected in amplifications of our samples.

\section{RESULTS}

\section{Milk, semen and urine cytological analysis}

Milk, urine and semen samples (Figure 1A) had high lymphocyte and epithelial-like cell counts, as well as signs of cell debris. While the frozen semen samples had the expected density of spermatozoa, the seminal fluid samples collected from strongly affected bulls were azoospermic. 

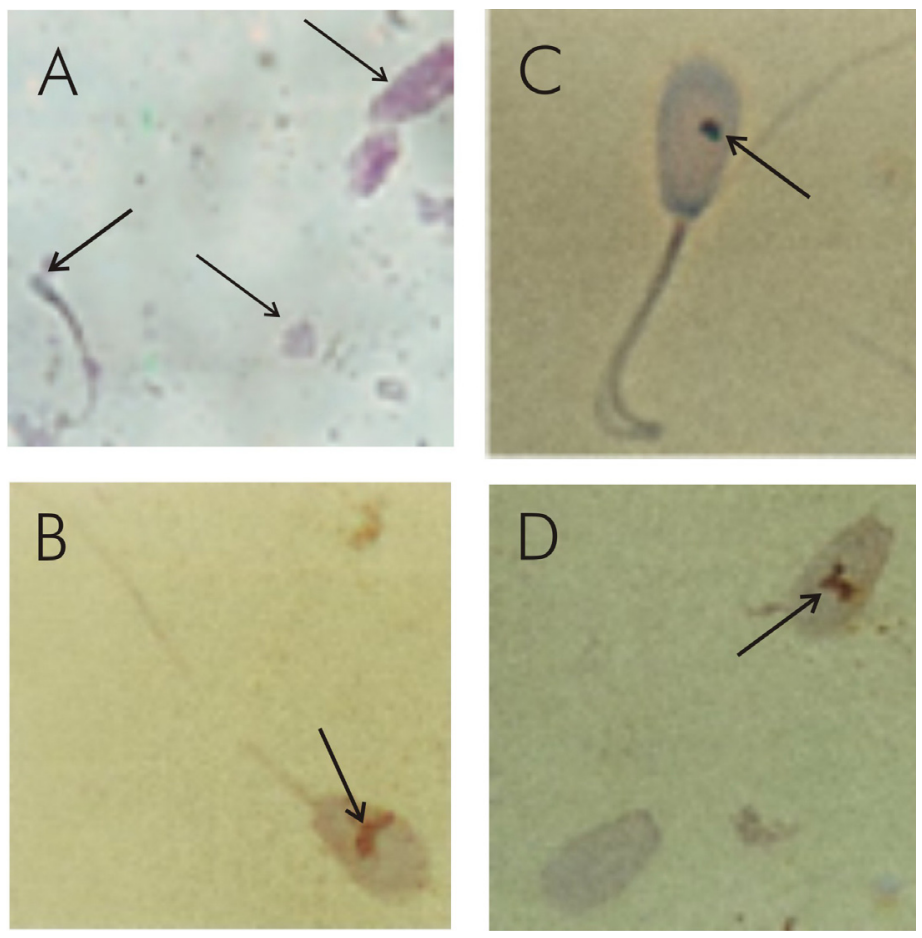

Figure 1. A. Giemsa-stained smear prepared from a frozen semen sample showing lymphocyte and epithelium-like cell debris (light arrows) and a spermatozoon (heavy arrow). B-D. In situ hybridization labeling of spermatozoids (arrows) with a probe for the L1 region of the bovine papillomavirus 2 gene.

\section{BPV DNA identification in tissues and body fluids}

Gene amplification by PCR with selective primer pairs directed to the L1, L2, and E7 regions of the BPV types was performed on total DNA extracted from tissue and body fluid samples from the BPV-infected cattle. Eighteen of the 21 milk samples had both BPV4 and BPV2 DNA, two had only BPV4 DNA and one sample had no BPV DNA. BPV1 was not detected in any of the milk samples (Table 2). Among the six urine samples, three showed amplification products corresponding to BPV2 and BPV4 DNA, two urine samples to one viral type and one to neither viral type. The 12 semen samples showed BPV2 DNA, but not BPV1 or BPV4 DNA. On the other hand, the three seminal fluid samples carried both BPV1 and BPV2 DNA. Eighty percent of the peripheral blood samples were found to carry BPV1 DNA, while $90 \%$ of the samples carried both BPV2 and BPV4 DNA. All eight wart tissue samples were found to have BPV1, BPV2 and BPV4 DNA (Table 2). Figure 2 shows examples of amplification products of BPV viral types in milk, semen, seminal fluid, urine, and blood of diseased animals. The amplified DNA products were cycle-sequenced and the nucleotide sequences were compared to published sequences of BPV types for identity confirmation. 
Homology of the identified DNA sequences was from 95 to $99 \%$ of respective BPV1, 2 and 4 haplotypes (Genebank). In situ hybridization of BPV2-positive semen samples using a biotinlabeled BPV2 DNA amplicon probe revealed intense staining in spermatozoa heads (Figure $1 \mathrm{~B}, \mathrm{C}$, and D).

\begin{tabular}{|c|c|c|c|c|}
\hline \multirow{2}{*}{$\begin{array}{l}\text { Samples } \\
\text { Tissue/fluid }\end{array}$} & \multicolumn{4}{|c|}{ Virus type } \\
\hline & $\mathrm{N}$ & BPV1 & BPV2 & BPV4 \\
\hline Milk & 21 & 0 & 18 & 20 \\
\hline Frozen semen & 12 & 0 & 12 & 0 \\
\hline Seminal fluid & 3 & 3 & 3 & 0 \\
\hline Urine & 6 & - & 4 & 3 \\
\hline Blood & 12 & 10 & 11 & 11 \\
\hline Wart & 8 & 8 & 8 & 8 \\
\hline Total & 62 & 21 & 56 & 42 \\
\hline
\end{tabular}
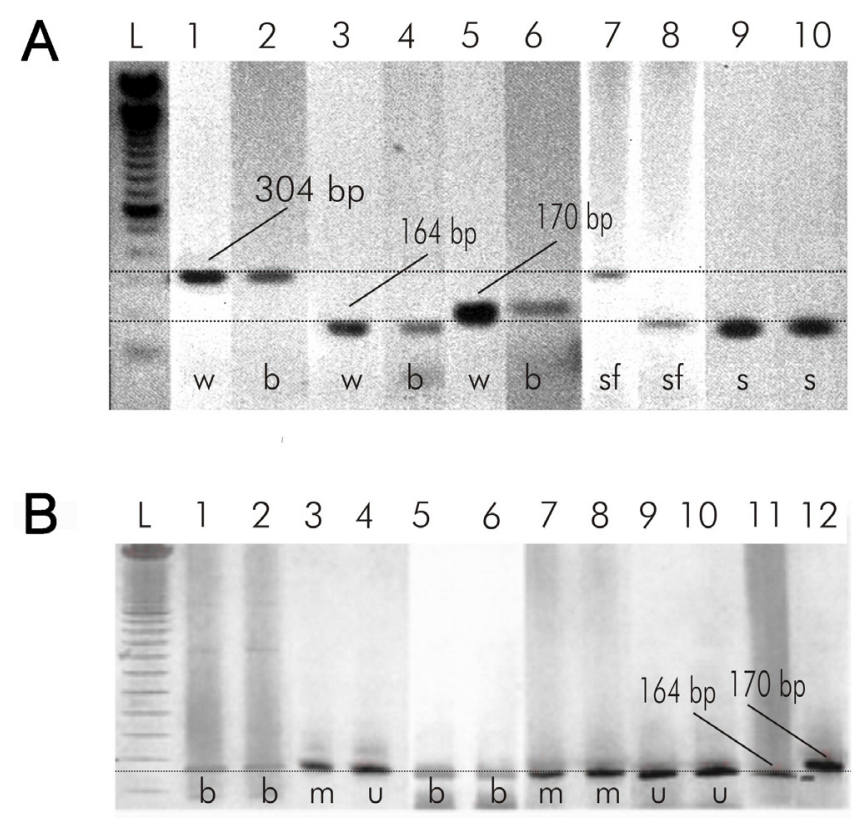

Figure 2. Amplification products of bovine papillomavirus (BPV) gene sequences from bovine milk (m), semen (s), seminal fluid (sf), urine (u), blood (b), and warts (w). The amplified DNA sequences correspond to the L2, L1 or E7 gene regions of BPV1, BPV2 or BPV4, respectively. A. BPV1 (301 bp), BPV2 (164 bp) and BPV4 (170 bp) DNA amplification products from wart (lanes 1,3,5) and blood samples (lanes 2, 4, 6), respectively; BPV1 and BPV2 in seminal fluid samples (lanes 7, 8); BPV2 amplification products in semen (lanes 9, 10). Ethidium bromide-stained $1 \%$ agarose gel. B. BPV4 and BPV2 in blood (lanes 1, 2, 5, 6), milk (lanes 3, 7, 8) or urine samples (lanes 4, 9, 10) samples, respectively; BPV2 and BPV 4 positive controls (lanes 11, 12). Silver-stained 12.5\% SDS-PAGE gel. L= 100-bp ladder molecular weight markers. 


\section{DISCUSSION}

Through gene amplification, we identified various BPV types in body fluids and tissues. The identity of these viruses was confirmed by homology of the amplified DNA products to published genome sequences of haplotype BPV DNA sequences. The detection of BPV1, 2 and 4 in milk, urine, seminal fluid, and sperm cells demonstrates that these viruses can be transmitted by various mechanisms other than physical contact of epithelia or contact with contaminated instruments. Suckling and sexual intercourse are the main means of direct body fluid exchange among cattle and the finding of BPV both in milk and seminal fluid indicate two important mechanisms of viral transmission. Additionally, the finding of BPV2 DNA in frozen semen samples obtained from an artificial insemination facility highlights the potential dissemination of papillomavirus-related diseases via artificial insemination. Contamination of pastures by urine-born papillomavirus may also be an important means of BPV transmission to cattle or equines. Equine sarcoidosis in horses is associated with BPV type 1 and 2 infections (Yuan et al., 2008; Brandt et al., 2008; Bogaert et al., 2008).

Bovine papillomavirus 2 has been previously described in peripheral blood (Stocco dos Santos et al., 1998; Wosiacki et al., 2005) and the high titer of BPV2 DNA that we found in lymphocytes confirms earlier predictions (Campo et al., 1994; Stocco dos Santos et al., 1993, 1998) and agrees with recent reports (Brandt et al., 2008) that lymphocytes can harbor papillomavirus and are latent sites of viral infection. In the other fluids, including milk, urine, and seminal fluid, papillomavirus may be in lymphocytes or exfoliated epithelial cells. Both were found in higher than usual numbers in BPV-infected animals. Peripheral blood mononuclear cells, related to inflammatory events in mammary glands, have been found in milk from animals infected with various different pathogens (Riollet et al., 2000; Leitner et al., 2000). Our findings of BPV DNA in peripheral blood lymphocytes demonstrate host immune system and virus interactions. Lymphocytes may not only provide latent sites of infection, but also may act as potential vectors of BPV dissemination both within and between animals.

It has been proposed that papillomavirus DNA is from fragments of viral breakdown products rather than functional viral genomes. However, the fact that BPV infection can be transmitted to recipient animals inoculated with blood from BPV2-infected cows (Stocco dos Santos et al., 1998) demonstrates competent BPV genome in peripheral blood. Additionally, naked DNA fragments would not be expected to survive whole-blood DNAse or macrophageactivity.

We need to consider the infectivity potential of BPV-contaminated milk, urine, semen, and sperm cells. Given that intra-muscularly injected lymphocyte-born BPV (Stocco dos Santos et al., 1998) and keratinocytes on contaminated instruments in contact with healthy epidermis (Jarrett, 1985) can transmit infection, it is clear that milk, urine and semen from infected animals can be BPV vectors. The detection of BPV2 DNA in spermatozoa adds a new dimension to what we know about viral transmission mechanisms. Infected spermatozoa could result in embryonic infection on conception, characterizing vertical transmission. The three heavily infected bulls from which seminal fluid was gathered were clinically azoospermic, suggesting that BPV directly affects sperm production by infecting gonad germ cells. Supporting the hypothesis of vertical papillomavirus transmission, BPV2 DNA was found in the blood of newborn calves, including those delivered by cesarean procedures. Intrauterine BPV transmission occurs, if not at conception, at some time during embryonic or fetal development. 
We found tissues and/or body fluids of individual animals to be infected by more than one viral type. Providing that the animals were not simultaneously infected by multiple viral types, this finding demonstrates that BPV infection by one virus type offers little or no protection against subsequent infection with other BPV types. We can now challenge the prevailing concept that papillomaviruses are exclusively epitheliotropic, as we found BPV DNA in most of the tissue types that we investigated. Along with epithelial cells, BPV viruses have been found in peripheral blood lymphocytes, urinary bladder neoplasic and vascular tissues (Campo et al., 1992; Wosiacki et al., 2005), as well as in spermatozoa and female reproductive tissues (Carvalho et al., 2003; Yaguiu et al., 2008). Lymphocytes are potential latency sites (Campo et al., 1994; Stocco dos Santos et al., 1993, 1998) for papillomavirus that can actively disseminate infective papilloma viral DNA within an organism to reach different tissues or act as carriers of the virus in body fluids, such as milk and semen. We recently found expression of BPV2 L1 capsid protein in lymphocyte cells (data not shown), implying that the biological cycle of the papillomavirus is completed in these cells. Given the ubiquity of multiple BPV types in body tissues and fluids and the multiplicity of vertical and horizontal transmission mechanisms that have been unveiled, sanitary and prophylactic measures against BPV transmission should include screening of semen banks and effective vaccination with polyvalent prophylactic and therapeutic vaccines.

\section{ACKNOWLEDGMENTS}

The authors thank the Conselho Nacional de Pesquisa e Desenvolvimento Tecnológico $(\mathrm{CNPq})$ and the Fundação de Amparo à Pesquisa do Estado de São Paulo (FAPESP), for grants and fellowships.

\section{REFERENCES}

Bogaert L, Martens A, Van Poucke M, Ducatelle R, et al. (2008). High prevalence of bovine papillomaviral DNA in the normal skin of equine sarcoid-affected and healthy horses. Vet. Microbiol. 129: 58-68.

Borzacchiello G and Roperto F (2008). Bovine papillomaviruses, papillomas and cancer in cattle. Vet. Res. 39: 45.

Brandt S, Haralambus R, Schoster A, Kirnbauer R, et al. (2008). Peripheral blood mononuclear cells represent a reservoir of bovine papillomavirus DNA in sarcoid-affected equines. J. Gen. Virol. 89: 1390-1395.

Campo MS (2003). Papillomavirus and disease in humans and animals. Vet. Comp. Oncol. 1: 3-14.

Campo MS, Jarrett WF, Barron R, O’Neil BW, et al. (1992). Association of bovine papillomavirus type 2 and bracken fern with bladder cancer in cattle. Cancer Res. 52: 6898-6904.

Campo MS, Jarrett WF, O'Neil W and Barron RJ (1994). Latent papillomavirus infection in cattle. Res. Vet. Sci. 56: 151-157.

Carvalho C, Freitas AC, Brunner O, Góes LGB, et al. (2003). Bovine papillomavirus type 2 in reproductive tract and gametes of slaughtered bovine females. Braz. J. Microbiol. 34 (Suppl 1): 82-84.

Jackson ME, Campo MS and Gaukroger JM (1993). Cooperation between papillomavirus and chemical cofactors in oncogenesis. Crit. Rev. Oncog. 4: 277-291.

Jarrett WF (1985). Bovine papillomaviruses. Clin. Dermatol. 3: 8-19.

Leitner G, Shoshani E, Krifucks O, Chaffer M, et al. (2000). Milk leucocyte population patterns in bovine udder infection of different aetiology. J. Vet. Med. B 47: 581-589.

Moura JW, Stocco dos Santos RC, Dagli ML, D'Angelino JL, et al. (1988). Chromosome aberrations in cattle raised on bracken fern pasture. Experientia 44: 785-788.

Olson C, Pamukcu AM, Brobst DF, Kowalczyk T, et al. (1959). A urinary bladder tumor induced by a bovine cutaneous papilloma agent. Cancer Res. 19: 779-782.

Olson C, Pamukcu AM and Brobst DF (1965). Papilloma-like virus from bovine urinary bladder tumors. Cancer 
Res. 25: 840-849.

Riollet C, Rainard P and Poutrel B (2000). Cells and cytokines in inflammatory secretions of bovine mammary gland. $A d v$. Exp. Med. Biol. 480: 247-258.

Stocco dos Santos RC, Ferraz OP, Castro NHC, Pinto JR, et al. (1993). Chromosome aberrations in cattle inoculated with blood of hematuric bovines. In: Seventeenth International Congress of Genetics, Birminghan.

Stocco dos Santos RC, Lindsey CJ, Ferraz OP, Pinto JR, et al. (1998). Bovine papillomavirus transmission and chromosomal aberrations: an experimental model. J. Gen. Virol. 79 (Pt 9): 2127-2135.

Wosiacki SR, Barreiros MAB, Alfieri AF and Alfieri AA (2005). Semi-nested-PCR for detection and typing of bovine papillomavirus type 2 in urinary bladder and whole blood from cattle with enzootic haematuria. J. Virol. Meth. 126: 215-219.

Yaguiu A, Carvalho C, Freitas AC, Góes LGB, et al. (2006). Papillomatosis in cattle: in situ detection of bovine papillomavirus DNA sequences in reproductive tissues. Braz. J. Morphol. Sci. 23: 129-136.

Yaguiu A, Dagli ML, Birgel EH Jr, Alves Reis BC, et al. (2008). Simultaneous presence of bovine papillomavirus and bovine leukemia virus in different bovine tissues: in situ hybridization and cytogenetic analysis. Genet. Mol. Res. 7: 487-497.

Yuan ZQ, Nicolson L, Marchetti B, Gault EA, et al. (2008). Transcriptional changes induced by bovine papillomavirus type 1 in equine fibroblasts. J. Virol. 82: 6481-6491. 